\title{
Propofol-cetamina racêmica e propofol-cetamina levógira em cadelas: parâmetros eletrocardiográficos e outras variáveis fisiológicas
}

\author{
[Propofol-racemic ketamine or propofol-levogire ketamine in dogs: effects on electrocardiography \\ and other physiological parameters] \\ R.M. Almeida ${ }^{1,2}$, C.E.V.Silva ${ }^{2}$, M. Zimmermann ${ }^{3}$, S. Maguilnik ${ }^{2}$ \\ ${ }^{1}$ Departamento de Medicina Veterinária - UPIS \\ SEPS 712/912, conjunto A, Asa Sul \\ 70390-125 - Brasília, DF \\ ${ }^{2}$ Universidade de Brasília - Brasília, DF \\ ${ }^{3}$ Médica veterinária autônoma
}

\begin{abstract}
RESUMO
Estudaram-se os efeitos da infusão contínua da associação propofol e cetamina sobre variáveis fisiológicas e eletrocardiográficas e sua possível analgesia em 12 cadelas. Após indução com propofol, os animais receberam $0,4 \mathrm{mg} / \mathrm{kg} / \mathrm{min}$ de propofol $+0,2 \mathrm{mg} / \mathrm{kg} / \mathrm{min}$ de cetamina racêmica $(\mathrm{n}=6$, grupo $\mathrm{PC}$ ) ou $0,4 \mathrm{mg} / \mathrm{kg} / \mathrm{min}$ de propofol $+0,1 \mathrm{mg} / \mathrm{kg} / \mathrm{min}$ de cetamina $\mathrm{S}+(\mathrm{n}=6$, grupo PCS $)$. Avaliaram-se: teste álgico, freqüência cardíaca (FC), parâmetros eletrocardiográficos, freqüência respiratória (FR), pressão arterial sistólica, média e diastólica (PAS, PAM, PAD), saturação da oxiemoglobina $\left(\mathrm{SpO}_{2}\right)$ e temperatura retal (TR). Houve elevação da FC sem alterações eletrocardiográficas, com exceção de aumento na amplitude da onda $\mathrm{T}$ em um animal de cada grupo. A FR diminuiu, e os valores de $\mathrm{SpO}_{2}$ ficaram abaixo de $90 \%$ em alguns momentos nos dois grupos. PAS, PAM e PAD diminuíram, mas não houve diferença entre os protocolos. Não se observou analgesia em sete animais, três cadelas apresentaram analgesia discreta, e apenas duas demonstraram analgesia favorável. Conclui-se que os protocolos são seguros em cadelas, contudo não há analgesia suficiente para procedimento cirúrgico. As alterações eletrocardiográficas foram relacionadas à $\mathrm{FC}$ e à amplitude de onda $\mathrm{T}$, sendo esta sugestiva de hipóxia do miocárdio.
\end{abstract}

Palavras-chave: cadela, propofol, cetamina, cetamina S+, eletrocardiografia

\begin{abstract}
The effects of propofol and ketamine on physiological parameters, electrocardiography, and analgesia were evaluated in twelve dogs that received propofol-ketamine $(0.4 \mathrm{mg} / \mathrm{kg} / \mathrm{min}+0.2 \mathrm{mg} / \mathrm{kg} / \mathrm{min}, \mathrm{n}=6, P K$ group) or propofol-S+ketamine $(0.4 \mathrm{mg} / \mathrm{kg} / \mathrm{min}+0.1 \mathrm{mg} / \mathrm{kg} / \mathrm{min}, \mathrm{n}=6$, , PKS group) after induction of anesthesia with propofol $(8.0 \mathrm{mg} / \mathrm{kg})$. Assessments of pain; heart rate (HR); electrocardiography (ECG); respiratory rate (RR); systolic, medium, and diastolic arterial pressures (SAP, MAP, DAP); saturation of hemoglobin $\left(\mathrm{SpO}_{2}\right)$; and rectal temperature (RT) were conducted. There was a rise in HR with no electrocardiographically changes, but an increase in amplitude of $T$ wave in one animal of each group. $R R$ decreased and $\mathrm{SpO}_{2}$ were lower than $90 \%$ in two moments of both groups. SAP, MAP, and DAP were reduced during the experimental period, but with no statistical difference between the protocols. There was no analgesia in seven bitches, mild analgesia was observed in three, and satisfactory analgesia in only two animals tested. It was concluded that the protocols are safe for dogs, but there is no analgesia for surgical procedures. The changes in ECG are related to HR and amplitude of $T$ wave, which may be due to myocardial hypoxia.
\end{abstract}

Keywords: dog, propofol, ketamine, S+ ketamine, electrocardiography

Recebido em 17 de março de 2008

Aceito em 28 de outubro de 2008

E-mail: ricardoalmeida@upis.br 


\section{INTRODUÇÃO}

O propofol, um anestésico geral fenólico, tem sido utilizado como agente indutor e de manutenção anestésica em diferentes espécies domésticas. Apresenta características desejáveis, como rápida indução, bons planos anestésicos e recuperação tranqüila, porém sua ação analgésica é classificada como fraca (Branson e Gross, 1994; Short e Bufalari, 1999).

A cetamina, um anestésico dissociativo com propriedades analgésicas, deve ser usada em associação com outros fármacos, pois pode causar estímulo cardiovascular, catalepsia e recuperação agitada (Wright, 1982; Haskins et al., 1985). O isômero levógiro $\mathrm{S}(+)$ da cetamina pode apresentar efeitos analgésico e anestésico de duas a quatro vezes mais intensos que o efeito da mistura racêmica, além de provocar menos reações desagradáveis na recuperação anestésica (Lauretti et al., 2000). Em cães, essa apresentação da cetamina apresentou maior potência anestésica e melhor recuperação, por isso sua dose deve ser reduzida em relação à forma racêmica (Duque et al., 2005).

Os objetivos desse estudo foram realizar anestesia total intravenosa por infusão contínua com a associação desses fármacos e avaliar a possível analgesia e os efeitos sobre as variáveis fisiológicas e eletrocardiográficas em cadelas.

\section{MATERIAL E MÉTODOS}

Utilizaram-se 12 cadelas, sem raça definida, com idades entre dois e quatro anos e pesos entre 7,5 e $18 \mathrm{~kg}$, consideradas sadias pela avaliação clínica e laboratorial. As cadelas permaneceram em canis, onde receberam ração duas vezes ao dia e água à vontade durante $\mathrm{o}$ período experimental. Foram submetidas a jejum de 12 horas para alimentos sólidos e de duas horas para água, anteriores ao experimento.

Dois grupos, de igual número, formados aleatoriamente, receberam como anestesia intravenosa $0.4 \mathrm{mg} / \mathrm{kg} / \mathrm{min}$ de propofol $^{1}$ associado a $0,2 \mathrm{mg} / \mathrm{kg} / \mathrm{min}$ de cetamina racêmica ${ }^{2}$ (PC), ou $0,4 \mathrm{mg} / \mathrm{kg} / \mathrm{min}$ de propofol associado a

${ }^{1}$ Diprivan 1\%, Astra Zeneca Farmacêutica - Cotia, Brasil ${ }^{2}$ Vetaset $10 \%$, Fort Dodge Saúde Animal Ltda. - Campinas, Brasil.
$0,1 \mathrm{mg} / \mathrm{kg} / \mathrm{min}$ de cetamina levógira ${ }^{3}$ (PCS). Todos os animais receberam, como indução anestésica, propofol na dose máxima de $8,0 \mathrm{mg} / \mathrm{kg}$ por via intravenosa lenta, sem prémedicação, através de um cateter ${ }^{4}$ introduzido na veia cefálica direita, até o relaxamento da mandíbula e a possibilidade de intubação orotraqueal. A administração contínua dos fármacos foi realizada após cinco minutos da indução, por meio de bomba infusora ${ }^{5}$, à taxa de infusão igual a $20 \mathrm{ml} / \mathrm{kg} / \mathrm{h}$, com o volume final completado com solução fisiológica ${ }^{6}$.

As cadelas foram examinadas antes dos tratamentos para a obtenção dos valores basais (M0). Avaliaram-se as seguintes variáveis eletrocardiográficas ${ }^{7}$ : freqüência cardíaca (FC), duração e amplitude da onda $\mathrm{P}$, duração do complexo QRS, amplitude da onda R, duração dos intervalos PR e QT, polaridade da onda T e segmento ST, registrando-se as três derivações bipolares (I, II, III) e as três derivações unipolares aumentadas (aVR, aVL, aVF), à velocidade de $50 \mathrm{~mm} / \mathrm{s}$ e sensibilidade $1 \mathrm{mv}=$ $1 \mathrm{~cm}$. Outras variáveis fisiológicas avaliadas foram: temperatura retal (TR), freqüência respiratória (FR), por meio de observação do movimento do tórax durante um minuto, pressões arteriais sistólica, média e diastólica (PAS, PAM, PAD) e saturação de oxigênio da hemoglobina $\left(\mathrm{SpO}_{2}\right)$, usando-se um monitor multiparamétrico ${ }^{8}$. Neste caso, o manguito de pressão foi posicionado no membro torácico esquerdo, na região do rádio/ulna, e o sensor de oximetria de pulso fixado na língua. Além disso, foi apreciada a possível analgesia pinçando-se a pele na região interdigital do membro pélvico esquerdo com pinça de Kocker, até no máximo a segunda trava, durante dois segundos. Os graus de analgesia foram classificados em: ausente resposta de retirada de membro antes ou na primeira trava -, discreta - retirada do membro na segunda trava -, e favorável - sem retirada do membro na segunda trava. Todas as avaliações

${ }^{3}$ Ketamin $\mathrm{S}(+) \quad 5 \%$ Cristália Produtos Químicos Farmacêuticos Ltda.- Itapira, Brasil.

${ }^{4}$ Cateter intravascular periférico Jelco $22 \mathrm{G}$, Johnson \& Johnson Ltda.- São José dos Campos, Brasil.

${ }^{5}$ Digibomb, Fundação Adib Jatene - São Paulo, Brasil.

${ }^{6}$ Solução isotônica de $\mathrm{NaCl}$ 0,9\%, Aster Produtos Médicos Sorocaba, Brasil.

${ }^{7}$ Monitor eletrocardiográfico TEB - C10, Tecnologia Eletrônica Brasileira Ltda.- São Paulo, Brasil.

${ }^{8}$ Monitor multiparamétrico Life window 6000, Digicare Biomedical Technology - Palm Beach, EUA. 
foram realizadas cinco minutos após a indução anestésica (M1), aos cinco e 10 minutos após o início da infusão contínua (M2 e M3) e a cada 10 minutos, até o total de 60 minutos da anestesia intravenosa (M4 a M8). O tempo de recuperação anestésica observado foi desde o instante final da infusão anestésica até a estação do animal.

Os dados referentes às variáveis fisiológicas e eletrocardiográficas foram avaliados por meio de análise de variância (ANOVA) para detecção de diferenças dos momentos entre os grupos, e por análise de variância para repetições múltiplas (ANOVA RM) para os momentos dentro de cada grupo. Os graus de analgesia entre os diferentes grupos foram analisados pelo teste de quiquadrado. $\mathrm{O}$ nível de significância para os testes estatísticos foi ajustado para $\mathrm{P} \leq 0,05$.

\section{RESULTADOS E DISCUSSÃO}

Os resultados dos parâmetros eletrocardiográficos e de outras variáveis fisiológicas são apresentados nas Tab. 1 e 2 .

Tabela 1. Valores médios e desvios-padrão de variáveis fisiológicas de cadelas tratadas com propofol e cetamina (PC) ou propofol e cetamina S(+) (PCS), segundo os tempos de avaliação

\begin{tabular}{|c|c|c|c|c|c|c|c|c|c|c|}
\hline \multirow{2}{*}{ Variável } & & \multicolumn{9}{|c|}{ Momento } \\
\hline & & 0 & 1 & 2 & 3 & 4 & 5 & 6 & 7 & 8 \\
\hline \multirow{4}{*}{$\begin{array}{c}\mathrm{FC} \\
(\mathrm{bpm})\end{array}$} & $\mathrm{PC}$ & 119,17 & 122,33 & 142,50 & 145,17 & 145,67 & 141,33 & 134,50 & 139,33 & 148,33 \\
\hline & & $\pm 24,17 \mathrm{aA}$ & $\pm 21,94 \mathrm{aA}$ & $\pm 8,02 \mathrm{bA}$ & $\pm 26,45 \mathrm{bA}$ & $\pm 36,02 \mathrm{bA}$ & $\pm 36,02 \mathrm{bA}$ & $\pm 40,5 \mathrm{bA}$ & $\pm 30,68 \mathrm{bA}$ & $\pm 38,62 \mathrm{bA}$ \\
\hline & PCS & 95,17 & 112,83 & 143,83 & 145,17 & 133,00 & 131,67 & 123,50 & 128,50 & 127,00 \\
\hline & & $\pm 18,14 \mathrm{aA}$ & $\pm 19,10 \mathrm{aA}$ & $\pm 32,20 \mathrm{bA}$ & $\pm 26,95 \mathrm{bA}$ & $\pm 22,62 \mathrm{bA}$ & $\pm 20,33 \mathrm{bA}$ & $\pm 21,75 \mathrm{bA}$ & $\pm 20,10 \mathrm{bA}$ & $\pm 18,25 \mathrm{bA}$ \\
\hline \multirow{4}{*}{$\begin{array}{c}\text { PAS } \\
(\mathrm{mmHg})\end{array}$} & $\mathrm{PC}$ & 133,67 & 110,67 & 111,00 & 103,17 & 100,50 & 102,67 & 105,83 & 110,16 & 112,83 \\
\hline & & $\pm 11,27 \mathrm{aA}$ & $\pm 13,09 \mathrm{bA}$ & $\pm 10,45 \mathrm{bA}$ & $\pm 10,61 \mathrm{bA}$ & $\pm 10,01 \mathrm{bA}$ & $\pm 16,43 \mathrm{bA}$ & $\pm 20,26 \mathrm{bA}$ & $\pm 16,18 \mathrm{bA}$ & $\pm 17,37 \mathrm{bA}$ \\
\hline & PCS & 133,67 & 115,00 & 111,50 & 112,00 & 106,50 & 108,33 & 111,33 & 112,50 & 116,33 \\
\hline & & $\pm 32,96 \mathrm{aA}$ & $\pm 21,32 \mathrm{bA}$ & $\pm 14,84 \mathrm{bA}$ & $\pm 17,72 \mathrm{bA}$ & $\pm 11,15 \mathrm{bA}$ & $\pm 13,97 \mathrm{bA}$ & $\pm 13,54 \mathrm{bA}$ & $\pm 17,35 \mathrm{bA}$ & $\pm 16,03 \mathrm{bA}$ \\
\hline \multirow{4}{*}{$\begin{array}{c}\text { PAM } \\
(\mathrm{mmHg})\end{array}$} & $\mathrm{PC}$ & 104,67 & 83,00 & 89,83 & 79,33 & 76,50 & 77,17 & 79,00 & 85,83 & 87,67 \\
\hline & & $\pm 12,11 \mathrm{aA}$ & $\pm 8,69 \mathrm{bA}$ & $\pm 14,30 \mathrm{bA}$ & $\pm 8,57 \mathrm{bA}$ & $\pm 6,66 \mathrm{bA}$ & $\pm 13,38 \mathrm{bA}$ & $\pm 16,73 \mathrm{bA}$ & $\pm 16,00 \mathrm{bA}$ & $\pm 16,74 \mathrm{bA}$ \\
\hline & PCS & 89,17 & 88,50 & 81,17 & 81,67 & 75,67 & 76,83 & 82,83 & 84,00 & 88,83 \\
\hline & & $\pm 11,44 \mathrm{aA}$ & $\pm 15,36 \mathrm{bA}$ & $\pm 12,89 \mathrm{bA}$ & $\pm 16,99 \mathrm{bA}$ & $\pm 11,25 \mathrm{bA}$ & $\pm 12,42 \mathrm{bA}$ & $\pm 17,00 \mathrm{bA}$ & $\pm 21,44 \mathrm{bA}$ & $\pm 18,79 \mathrm{bA}$ \\
\hline \multirow{4}{*}{$\begin{array}{c}\text { PAD } \\
(\mathrm{mmHg})\end{array}$} & $\mathrm{PC}$ & 83,33 & 63,50 & 74,50 & 60,50 & 62,17 & 65,50 & 63,00 & 74,17 & 71,50 \\
\hline & & $\pm 10,98 \mathrm{aA}$ &, $\pm 50 \mathrm{bA}$ & $\pm 19,88 \mathrm{bA}$ & $\pm 10,99 \mathrm{bA}$ & $\pm 6,34 \mathrm{bA}$ & $\pm 11,96 \mathrm{bA}$ & $\pm 11,98 \mathrm{bA}$ & $\pm 14,51 \mathrm{bA}$ & $\pm 12,21 \mathrm{bA}$ \\
\hline & PCS & 67,33 & 72,33 & 61,50 & 62,00 & 57,17 & 58,17 & 64,83 & 67,33 & 72,50 \\
\hline & & $\pm 16,78 \mathrm{aA}$ & $\pm 15,54 \mathrm{bA}$ & $\pm 13,62 \mathrm{bA}$ & $\pm 13,87 \mathrm{bA}$ & $\pm 12,25 \mathrm{bA}$ & $\pm 12,46 \mathrm{bA}$ & $\pm 16,70 \mathrm{bA}$ & $\pm 21,90 \mathrm{bA}$ & $\pm 20,37 \mathrm{bA}$ \\
\hline \multirow{4}{*}{$\begin{array}{c}\text { FR } \\
(\mathrm{mpm})\end{array}$} & $\mathrm{PC}$ & 25,00 & 18,67 & 18,00 & 17,83 & 17,67 & 12,67 & 13,67 & 12,00 & 15,00 \\
\hline & & $\pm 4,15 \mathrm{aA}$ & $\pm 9,18 \mathrm{aA}$ & $\pm 11,66 \mathrm{bA}$ & $\pm 9,93 \mathrm{bA}$ & $\pm 9,16 \mathrm{bA}$ & $\pm 3,72 \mathrm{bA}$ & $\pm 4,63 \mathrm{bA}$ & $\pm 2,83 \mathrm{bA}$ & $\pm 3,52 \mathrm{bA}$ \\
\hline & PCS & 33,00 & 35,00 & 40,33 & 30,83 & 19,67 & 22,00 & 18,33 & 19,50 & 19,67 \\
\hline & & $\pm 16,33 \mathrm{aA}$ & $\pm 10,18 \mathrm{aA}$ & $\pm 21,70 \mathrm{aA}$ & $\pm 15,88 \mathrm{aA}$ & $\pm 3,14 \mathrm{bA}$ & $\pm 5,93 \mathrm{bA}$ & $\pm 2,42 \mathrm{bA}$ & $\pm 3,45 \mathrm{bA}$ & $\pm 5,09 \mathrm{bA}$ \\
\hline \multirow{4}{*}{$\begin{array}{c}\mathrm{SpO}_{2} \\
(\%)\end{array}$} & $\mathrm{PC}$ & 98,16 & 94,16 & 87,50 & 87,33 & 92,83 & 94,67 & 95,00 & 95,67 & 94,00 \\
\hline & & $\pm 0,94 \mathrm{aA}$ & $\pm 0,87 \mathrm{aA}$ & $\pm 1,45 \mathrm{bA}$ & $\pm 2,21 \mathrm{bA}$ & $\pm 2,35 \mathrm{aA}$ & $\pm 0,37 \mathrm{aA}$ & $\pm 1,37 \mathrm{aA}$ & $\pm 2,85 \mathrm{aA}$ & $\pm 2,54 \mathrm{aA}$ \\
\hline & PCS & 93,67 & 94,33 & 92,16 & 90,17 & 90,17 & 93,50 & 91,50 & 92,67 & 93,17 \\
\hline & & $\pm 0,90 \mathrm{aA}$ & $\pm 1,22 \mathrm{aA}$ & $\pm 1,32 \mathrm{aB}$ & $\pm 0,99 \mathrm{bA}$ & $\pm 1,67 \mathrm{bB}$ & $\pm 2,14 \mathrm{aA}$ & $\pm 1,22 \mathrm{aA}$ & $\pm 0,92 \mathrm{aA}$ & $\pm 0,95 \mathrm{aA}$ \\
\hline \multirow{4}{*}{$\begin{array}{c}\mathrm{TR} \\
\left({ }^{\circ} \mathrm{C}\right)\end{array}$} & $\mathrm{PC}$ & 38,87 & 38,38 & 38,31 & 37,97 & 37,80 & 37,52 & 37,25 & 37,08 & 37,02 \\
\hline & & $\pm 0,45 \mathrm{aA}$ & $\pm 0,45 \mathrm{aA}$ & $\pm 0,44 \mathrm{aA}$ & $\pm 0,35 \mathrm{aA}$ & $\pm 0,44 \mathrm{aA}$ & $\pm 0,47 \mathrm{aA}$ & $\pm 0,62 \mathrm{aA}$ & $\pm 0,65 \mathrm{aA}$ & $\pm 0,65 \mathrm{aA}$ \\
\hline & PCS & 38,78 & 38,28 & 38,12 & 37,90 & 37,65 & 37,45 & 37,20 & 37,05 & 36,88 \\
\hline & & $\pm 0,37 \mathrm{aA}$ & $\pm 0,39 \mathrm{aA}$ & $\pm 0,36 \mathrm{aA}$ & $\pm 0,38 \mathrm{aA}$ & $\pm 0,34 \mathrm{aA}$ & $\pm 0,43 \mathrm{aA}$ & $\pm 0,48 \mathrm{aA}$ & $\pm 0,52 \mathrm{aA}$ & $\pm 0,54 \mathrm{aA}$ \\
\hline
\end{tabular}

Dentro dos grupos, letras minúsculas distintas indicam diferenças $(\mathrm{P}<0,05)$.

Entre os grupos, letras maiúsculas distintas indicam diferenças $(\mathrm{P}<0,05)$.

FC: freqüência cardíaca; PAS: pressão arterial sistólica; PAM: pressão arterial média; PAD: pressão arterial diastólica; FR: freqüência respiratória; $\mathrm{SpO}_{2}$ : saturação da oxiemoglobina; TR: temperatura retal.

M0: antes dos tratamentos; M1: cinco minutos após a indução anestésica; M2 e M3: cinco e 10 minutos após a infusão contínua; M4 a M8: a cada 10 minutos após M3. 
Propofol-cetamina racêmica...

Tabela 2. Valores médios e desvios-padrão de parâmetros eletrocardiográficos* de cadelas tratadas com propofol e cetamina (PC) ou propofol e cetamina S(+) (PCS), segundo os tempos de avaliação

\begin{tabular}{|c|c|c|c|c|c|c|c|c|c|c|}
\hline \multirow{2}{*}{\multicolumn{2}{|c|}{ Variável }} & \multicolumn{9}{|c|}{ Momento } \\
\hline & & \multirow{3}{*}{$\begin{array}{c}0 \\
0,0489 \\
\pm 0,004\end{array}$} & \multirow{3}{*}{$\begin{array}{c}1 \\
0,0486 \\
\pm 0,003\end{array}$} & \multirow{3}{*}{$\begin{array}{c}2 \\
0,0486 \\
\pm 0,004\end{array}$} & \multirow{3}{*}{$\begin{array}{c}3 \\
0,0486 \\
\pm 0,005\end{array}$} & \multirow{3}{*}{$\begin{array}{c}4 \\
0,0486 \\
\pm 0,007\end{array}$} & \multirow{3}{*}{$\begin{array}{c}5 \\
0,0474 \\
\pm 0,003\end{array}$} & \multirow{3}{*}{$\begin{array}{c}6 \\
0,0466 \\
\pm 0,004\end{array}$} & \multirow{3}{*}{$\begin{array}{c}7 \\
0,0485 \\
\pm 0,005\end{array}$} & \multirow{3}{*}{$\begin{array}{c}8 \\
0,0467 \\
\pm 0,003\end{array}$} \\
\hline $\mathrm{P}$ & $\mathrm{PC}$ & & & & & & & & & \\
\hline (seg) & & & & & & & & & & \\
\hline & PCS & 0,0407 & 0,0417 & 0,0390 & 0,0427 & 0,0433 & 0,0421 & 0,0426 & 0,0410 & 0,0433 \\
\hline & & $\pm 0,004$ & $\pm 0,008$ & $\pm 0,002$ & $\pm 0,004$ & $\pm 0,007$ & $\pm 0,005$ & $\pm 0,003$ & $\pm 0,002$ & $\pm 0,006$ \\
\hline \multirow{4}{*}{$\begin{array}{c}\mathrm{P} \\
(\mathrm{mV})\end{array}$} & $\mathrm{PC}$ & 0,204 & 0,206 & 0,212 & 0,206 & 0,184 & 0,202 & 0,176 & 0,205 & 0,190 \\
\hline & & $\pm 0,032$ & $\pm 0,032$ & $\pm 0,033$ & $\pm 0,025$ & $\pm 0,030$ & $\pm 0,033$ & $\pm 0,032$ & $\pm 0,040$ & $\pm 0,042$ \\
\hline & PCS & 0,132 & 0,147 & 0,160 & 0,167 & 0,180 & 0,168 & 0,147 & 0,143 & 0,143 \\
\hline & & $\pm 0,047$ & $\pm 0,063$ & $\pm 0,056$ & $\pm 0,038$ & $\pm 0,036$ & $\pm 0,046$ & $\pm 0,051$ & $\pm 0,050$ & $\pm 0,058$ \\
\hline \multirow{4}{*}{$\begin{array}{c}\text { PR } \\
(\text { seg) }\end{array}$} & $\mathrm{PC}$ & 0,098 & 0,098 & 0,096 & 0,090 & 0,088 & 0,090 & 0,092 & 0,097 & 0,090 \\
\hline & & $\pm 0,011$ & $\pm 0,010$ & $\pm 0,011$ & $\pm 0,014$ & $\pm 0,015$ & $\pm 0,014$ & $\pm 0,016$ & $\pm 0,007$ & $\pm 0,014$ \\
\hline & PCS & 0,088 & 0,087 & 0,077 & 0,085 & 0,085 & 0,088 & 0,089 & 0,087 & 0,086 \\
\hline & & $\pm 0,007$ & $\pm 0,017$ & $\pm 0,007$ & $\pm 0,012$ & $\pm 0,015$ & $\pm 0,008$ & $\pm 0,005$ & $\pm 0,005$ & $\pm 0,007$ \\
\hline \multirow{4}{*}{$\begin{array}{l}\text { QRS } \\
\text { (seg) }\end{array}$} & $\mathrm{PC}$ & 0,061 & 0,060 & 0,061 & 0,061 & 0,059 & 0,058 & 0,059 & 0,058 & 0,059 \\
\hline & & $\pm 0,004$ & $\pm 0,008$ & $\pm 0,008$ & $\pm 0,006$ & $\pm 0,003$ & $\pm 0,005$ & $\pm 0,004$ & $\pm 0,006$ & $\pm 0,006$ \\
\hline & PCS & 0,057 & 0,060 & 0,057 & 0,060 & 0,60 & 0,058 & 0,059 & 0,060 & 0,059 \\
\hline & & $\pm 0,005$ & $\pm 0,005$ & $\pm 0,008$ & $\pm 0,005$ & $\pm 0,004$ & $\pm 0,006$ & $\pm 0,004$ & $\pm 0,005$ & $\pm 0,005$ \\
\hline \multirow{4}{*}{$\begin{array}{c}\mathrm{R} \\
(\mathrm{mV})\end{array}$} & $\mathrm{PC}$ & 1,158 & 1,066 & 1,060 & 1,046 & 1,024 & 1,068 & 1,072 & 1,137 & 1,110 \\
\hline & & $\pm 0,289$ & $\pm 0,167$ & $\pm 0,187$ & $\pm 0,173$ & $\pm 0,144$ & $\pm 0,173$ & $\pm 0,181$ & $\pm 0,193$ & $\pm 0,193$ \\
\hline & PCS & 0,980 & 0,918 & 0,787 & 0,828 & 0,805 & 0,858 & 0,837 & 0,813 & 0,835 \\
\hline & & $\pm 0,419$ & $\pm 0,470$ & $\pm 0,491$ & $\pm 0,491$ & $\pm 0,487$ & $\pm 0,446$ & $\pm 0,447$ & $\pm 0,417$ & $\pm 0,445$ \\
\hline \multirow{4}{*}{$\begin{array}{c}\text { QT } \\
\text { (seg) }\end{array}$} & $\mathrm{PC}$ & 0,201 & 0,216 & 0,204 & 0,207 & 0,206 & 0,214 & 0,221 & 0,219 & 0,212 \\
\hline & & $\pm 0,013$ & $\pm 0,011$ & $\pm 0,017$ & $\pm 0,019$ & $\pm 0,020$ & $\pm 0,030$ & $\pm 0,035$ & $\pm 0,020$ & $\pm 0,030$ \\
\hline & PCS & 0,199 & 0,217 & 0,198 & 0,206 & 0,212 & 0,212 & 0,223 & 0,220 & 0,215 \\
\hline & & $\pm 0,021$ & $\pm 0,019$ & $\pm 0,022$ & $\pm 0,024$ & $\pm 0,021$ & $\pm 0,022$ & $\pm 0,025$ & $\pm 0,025$ & $\pm 0,027$ \\
\hline
\end{tabular}

* - Derivação II; velocidade $50 \mathrm{~mm} / \mathrm{s}$. Não houve diferença entre valores observados dentro de grupos e entre grupos.

No tratamento PC, houve elevação da FC do M2 até M8, ao passo que, no PCS, a FC aumentou em M2 e diminuiu em M4 até o fim das aferições, mantendo-se sempre acima de M0. Os valores de FC no grupo PCS sempre foram mais baixos que no grupo PC, a partir do M3. Em ambos os tratamentos, os valores de $\mathrm{FC}$ não retornaram aos basais até o fim do procedimento experimental. Nos dois tratamentos, ocorreu redução de PAS, PAM e PAD a partir de M1 até M8, sem diferença significativa entre os grupos.

De acordo com Smith et al. (1993), o efeito do propofol sobre o sistema cardiovascular é caracterizado por reduções do inotropismo, do débito cardíaco e da resistência periférica, levando à diminuição da PAM. A FC pode manter-se estável, demonstrando que o propofol pode inibir a resposta barorreflexa à hipotensão, como visto após a indução anestésica (Glowaski e Wetmore, 1999; Short e Bufalari, 1999). Em relação à manutenção anestésica, o propofol pode provocar diminuição dose-dependente da PAM (Aguiar et al., 1999), e a cetamina, por suas propriedades simpatomiméticas, pode elevar a FC e a PAM em animais sadios, sendo esta uma das razões de sua inclusão na anestesia intravenosa por infusão, com a finalidade de minimizar a depressão cardiovascular do propofol (Wright, 1982; Haskins et al., 1985; Seliskar et al., 1999; Intelizano et al., 2002; Selmi et al., 2005).

Segundo Vieira (1999), a cetamina não é capaz de prevenir a hipotensão produzida pelo propofol, apesar de ocorrer aumento da FC, o que pode ser atribuído a uma provável diminuição da resistência periférica ocasionada 
pelo propofol, como foi observado por Nishimori et al. (2006), ao usarem propofol por infusão contínua em taxa semelhante ao utilizado neste estudo. Wright (1982) relatou que a resposta vasopressora da cetamina também é mediada centralmente, em resposta à diminuição da atividade dos barorreceptores, mecanismo que pode ser inibido na presença de anestésicos gerais.

Segundo Muir e Hubbell (1988), Short e Bufalari (1999) e Glowaski e Wetmore (1999), a cetamina e o propofol deprimem de forma dosedependente os centros respiratórios superiores, podendo causar hipóxia. Do mesmo modo, Vainio (1991) e Duque et al. (2006) verificaram a diminuição dos valores de $\mathrm{FR}$ e $\mathrm{PaO}_{2}$ em cães anestesiados por infusões contínuas de propofol e cetamina, respectivamente. Similarmente ao ocorrido nos estudos citados, a FR diminuiu a partir de M2 em PC, e de M4 em PCS, e a $\mathrm{SpO}_{2}$ diminuiu de $90 \%$ em M2 e M3 em ambos os tratamentos, caracterizando um quadro de hipoventilação e hipóxia dos animais, reforçando assim a necessidade da suplementação de oxigênio durante o procedimento anestésico.

A TR não foi diferente entre os grupos e dentro deles, apesar de ter havido uma tendência à diminuição durante o procedimento anestésico, resultado provável de vasodilatação periférica e depressão dos centros termorregulatórios pelo propofol (Selmi et al., 2005).

Não houve ocorrências de arritmias de qualquer tipo, diferente do observado por Smith et al. (1993), que relataram episódios de extra-sístoles ventriculares após indução anestésica com propofol, e por Kamibayashi et al. (1991) e Paiva et al. (2002), que descreveram arritmias e bradiarritmias com o uso desse agente em cães.

Não houve diferenças na duração das ondas e dos intervalos entre os diferentes momentos em relação aos respectivos valores basais, com exceção do intervalo R-R, que diminuiu devido ao aumento da FC nos dois grupos experimentais, confirmando os resultados já apresentados por Souza et al. (2002), os quais demonstraram a mesma alteração eletrocardiográfica associada ao efeito simpatomimético da cetamina.
Quanto às amplitudes, as ondas $\mathrm{P}$ e $\mathrm{R}$ não diferiram dos valores iniciais, no entanto, ocorreu aumento significativo na amplitude da onda $\mathrm{T}$ ( $>25 \%$ da onda $\mathrm{R}$ ) em um animal de cada grupo, a partir de M2 até M8. De acordo com Tilley (1992), essa última alteração pode estar associada à isquemia ou hipóxia miocárdica e, no caso deste estudo, provavelmente essa alteração eletrocardiográfica está ligada à depressão da ventilação nos pacientes que ocorreu a partir dos mesmos tempos de aferição, associada ao fato de os animais não terem recebido complemento de oxigênio durante a anestesia intravenosa.

A associação cetamina-propofol tem como principal objetivo a promoção de analgesia, pois o propofol apresenta fraca ação analgésica (Haskins et al., 1985; Branson e Gross, 1994; Seliskar et al., 1999). Quanto ao escore do teste álgico, os grupos não diferiram entre si ou durante o período de observação, sendo que sete animais não demonstraram analgesia (escore zero), três apresentaram analgesia discreta (escore um) e apenas duas cadelas evidenciaram analgesia favorável (escore dois). Apesar de os animais com escore dois não demonstrarem reflexo de retirada do membro, observou-se que a FC aumentou após o pinçamento do interdígito, demonstrando que embora a analgesia seja favorável, o estímulo doloroso desencadeou uma resposta adrenérgica e, portanto, o protocolo não se mostrou indicado para a realização de qualquer intervenção cirúrgica, confirmando os resultados já descritos por Aguiar et al. (1999) e Intelizano et al. (2002). Com isso, o uso de um fármaco pré-anestésico que forneça analgesia adequada, ou um aumento da dose de cetamina faz-se necessário, já que o incremento da dose de propofol poderia aumentar o grau de depressão cardiovascular.

Em relação à recuperação pós-anestésica, Glowaski e Wetmore (1999) explicaram que a metabolização do propofol é realizada de maneira rápida, extra e intra-hepática. Vainio (1991), após administrar propofol para a indução e manutenção anestésicas de cães, relatou recuperação anestésica rápida e ausência de reações adversas. Resultados semelhantes foram observados por Watkins e Clarke (1987) e Morgan e Legge (1989), depois da indução de cães e gatos. Neste estudo, todos os animais mostraram recuperação tranqüila e rápida, sem complicações; o tempo de extubação foi de 
$6,5 \pm 3,7$ minutos no grupo $\mathrm{PC}$, e de 4,3 $\pm 2,9$ minutos no grupo PCS. As cadelas atingiram a posição quadrupedal aos $31 \pm 16,7$ e $14,3 \pm 2,6$ minutos para PC e PCS, respectivamente, após o término da infusão dos anestésicos. Não houve diferença entre os tratamentos em nenhum dos tempos relacionados à recuperação dos animais, apesar de o retorno anestésico ter sido mais rápido nos animais do grupo PCS. Lauretti et al. (2000) e Duque et al. (2005) demonstraram que a forma levógira da cetamina tem índices de reações desagradáveis mais baixos na recuperação anestésica em humanos e em cães, e que a dose pode ser diminuída em até $30 \%$ da dose da apresentação racêmica, em cães. No presente estudo, a redução da dose foi de $50 \%$, o que pôde ter influenciado discretamente o retorno à estação dos animais do grupo PCS.

\section{CONCLUSÕES}

Os protocolos são seguros para cadelas, apesar da discreta diminuição da pressão arterial, contudo não há analgesia suficiente para procedimento cirúrgico, sendo necessária a complementação analgésica. As alterações eletrocardiográficas de ambos os protocolos estão relacionadas à $\mathrm{FC}$ e possivelmente à amplitude de onda $\mathrm{T}$, sendo esta sugestiva de hipóxia do miocárdio, portanto tais protocolos anestésicos devem ser utilizados com precaução em pacientes cardiopatas. Adicionalmente, a presença de hipoventilação e hipóxia reforça a necessidade de suplementação de oxigênio durante todo o procedimento anestésico.

\section{REFERÊNCIAS BIBLIOGRÁFICAS}

AGUIAR, A.J.A.; LUNA, S.P.L.; OLIVA, V.N.L.S. et al. Continuous infusion of propofol in dogs premedicated with methotrimeprazine. J. Vet. Anaesth., v.26, p.38, 1999.

BRANSON, K.R.; GROSS, M.E. Propofol in veterinary medicine. J. Am. Vet. Med. Assoc., v.204, p.1888-1890, 1994.

DUQUE, J.C.M.; GUIRRO, E.C.B.; OLESKOVICZ, $\mathrm{N}$. et al. Determinação da potência relativa da cetamina S+ em cães. In: ENCONTRO DE ANESTESIOLOGIA VETERINÁRIA, 7., 2005, São Luís. Anais... São Luís: [s.n.] 2005. p.157. (Resumo).

DUQUE, J.C.M.; GUIRRO, E.C.B.; DUQUE, C.T.N. et al. Cardiorespiratory effects of ketamine or $\mathrm{S}(+)$ ketamine in sevoflurane anesthetized dogs undergoing to experimental hypovolemia. In: WORLD CONGRESS OF VETERINARY ANAESTHESIA, 9., 2006, Santos. Proceedings... Santos: [s.n.] 2006. p.188. (Abstract).

GLOWASKI, M.M.; WETMORE, L.A. Propofol: application in veterinary sedation and anesthesia. Clin. Tech. Small Anim. Pract., v.14, p.1-9, 1999.

HASKINS, S.C.; FARVER, T.B.; PATZ, J.D. Ketamine in dogs. Am. J. Vet. Res., v.46, p.1855-1860, 1985.

INTELIZANO, T.R.; CORTOPASSI, S.R.G.; KITAHARA, F.R. et al., Efeitos hemodinâmicos da anestesia total injetável com propofol, propofolquetamina racêmica e propofol-quetamina-s em cães. Rev. Bras. Cienc. Vet., v.9, supl., p.140-142, 2002.

KAMIBAYASHI, T.; HAYASHI, Y.; SUMIKAWA, $\mathrm{K}$ et al. Enhancement by propofol of epinephrineinduced arrhythmias in dogs. Anesthesiology, v.75, p.1035-1040, 1991.

LAURETTI, G.R.; LIMA, I.C.P.R.; BUSCATTI, R.Y. et al. Avaliação clínica dos efeitos hemodinâmicos, analgésicos, psicodélicos e do bloqueio muscular da cetamina racêmica e de seu $\mathrm{S}(+)$ isômero. Rev. Bras. Anestesiol., v.50, p.357-362, 2000.

MORGAN, D.W.T.; LEGGE, K. Clinical evaluation of propofol as an intravenous anaesthetic agent in cats and dogs. Vet. Rec., v.124, p.31-33, 1989.

MUIR III, W.W.; HUBBELL, J.A.E. Cardiopulmonary and anesthesic effects of ketamine and its enantiomers in dogs. Am. J. Vet. Res., v.49, p.530-533, 1988.

NISHIMORI, C.T.D.; NUNES, N.; PAULA, D.P. et al. Effects of three infusion rates of propofol with or without nitrous oxide on systemic and on cerebral hemodynamics in dogs. In: WORLD CONGRESS OF VETERINARY ANAESTHESIA, 9., 2006, Santos. Proceedings... Santos: [s.n.] 2006. p.187. (Abstract).

PAIVA, J.P.; MÁRSICO-FILHO, F.; RAMADINHA, R.H.R. et al. Alterações eletrocardiográficas durante a intubação endotraqueal em cães induzidos anestesiologicamente com tiopental sódico, propofol ou etomidato, com e sem pré-oxigenação. Rev. Bras. Cienc. Vet., v.9, supl., p.325-327, 2002.

SELISKAR, A.; BUTINAR, J.; PODGORNIK, D Propofol/ketamine versus propofol total intravenous anaesthesia in dogs: comparison and evaluation. J. Vet. Anaesth., v.26, p.43, 1999.

SELMI, A.L.; FIGUEIREDO, J.P.; MENDES, G.M. et al. Infusão contínua de propofol em gatos prémedicados com cetamina-midazolam. Arq. Bras. Med. Vet. Zootec., v.57, p.295-299, 2005. 
SHORT, C.E.; BUFALARI, A. Propofol anesthesia. Vet. Clin. N. Am.: Small Anim. Pract., v.29, p.747777, 1999.

SMITH, J.A.; GAYNOR, J.S.; BEDNARSKI, R.M. et al. Adverse effects of administration of propofol with various preanesthetic regimens in dogs. J. Am. Vet. Med. Assoc., v.202, p.1111-1115, 1993.

SOUZA, A.P.; CARARETO, R.; NUNES, N. et al. Eletrocardiografia em cães anestesiados com cetamina-s ou cetamina. Cienc. Rural, v.32, p.787791, 2002.

TILLEY, L.P. Essentials of canine and feline electrocardiography. Philadelphia: Lea \& Febiger, 1992. 470p

VAINIO, O. Propofol infusion anaesthesia in dogs pre-medicated with medetomidine. J. Vet. Anaesth., v.18, p.35-37, 1991.
VIEIRA, F.A.F. Comparação entre propofol $e$ propofol/quetamina na indução e manutenção anestésicas por infusão contínua intravenosa após medicação pré-anestésica com levomepronazina em cães. 1999. 123f. Dissertação (Mestrado) - Faculdade de Medicina Veterinária e Zootecnia, Universidade Estadual Paulista, Botucatu.

WATKINS, S.B.; CLARKE, K.W. Propofol as na intravenous anaesthetic agent in dogs. Vet. Rec.,v.120, p.326-329, 1987.

WRIGHT, M. Pharmacology effects of ketamine and its use in Veterinary medicine. J. Am. Vet. Med. Assoc., v.180, p.1462-1471, 1982. 